\title{
The Effects of Early Admission of Pregnant Women During Latent Phase on Pregnant Outcomes in Tabriz Taleghani Hospital
}

\author{
Somayyeh Naghi Zadeh ${ }^{1}$, Fahimeh Sehhatie Shafaie ${ }^{2 *}$, Morteza Ghojazadeh ${ }^{3}$
}

\begin{abstract}
Objective: The impossibility of determining the exact time of childbirth and measuring the length of latent phase may be the reason of different encounters with this stage of labor. This study investigates the impact of early admission of women in latent phase referred to Tabriz Taleghani Hospital.

Materials and Methods: A descriptive study was conducted in 2011 on 250 women during latent phase admitted to Tabriz Taleghani Hospital in which they were randomly selected for the survey. A five part questionnaire was used for data collection aimed to investigate (respectively) demographic characteristics, Partograph form, evaluating checklists of second, third and forth stages of labor, the results of a pre-discharge examination and also the first ten days after childbirth

Results: According to the findings $187 \mathrm{o}$

$\mathrm{f}$ the labors were stimulated by oxytocin induction (74.8\%) while $18.8 \%$ of the oxytocin treated women were hypotonic, 139 (55.6\%) underwent amniotomy, $6.8 \%$ of the fetus had FHR abnormalities including Bradicardia and tachycardia. No neonatal deaths were reported and just 2 of the newborns were admitted to the neonatal ward. Of the total $28(11.2 \%)$ had prolonged latent phase and 54 (21.6\%) of the patients had prolonged active phase of labor. The mean drop in hemoglobin and hematocrit rates during admission for delivery was, respectively, $1.35 \pm 1.41$ and $3.8 \pm 4.1$.

Conclusion: Admission in the latent phase of labor is associated with increased length of labor and stay in postnatal ward, increased maternal and infant complications and interventions. Therefore it is recommended that all the women should be given adequate training during pregnancy. They also should be advised that in the absence of medical problems it is best to be admitted when active labor begins to prevent many complications .

Keywords: Early Admission, Latent Phase, Pregnancy Outcomes
\end{abstract}

\section{Introduction}

Labor is defined as uterine contractions of sufficient frequency and duration and intensity to bring about demonstrable effacement and dilation of cervix (1). But this definition does not helpful in determining the actual onset of labor. The major obstacle to recognize a normal labor pattern is to determine the onset of it. However this can be done using several methods. The first method defines onset as the clock time when painful contractions come at regular intervals. In the second method beginning of the labor is defined as admission to labor unit. In National Maternity Hospital in Dublin the criteria for hospital admission of pregnant women includes painful contractions of the uterus. According to the criteria listed having painful hospitalization is necessary if contractions presenting with each of the following symptoms 1) ruptured membranes 2) bloody show 3) complete cervical effacement. In the USA all admission decisions are made based on cervical dilation rate (at least $3-4 \mathrm{~cm}$ ) with painful contractions (2).

According to Friedman (1972) the onset of labor is defined as that time at which the patient first perceived regular uterine contractions. The latent phase ends when the cervix is $3-5 \mathrm{~cm}$ dilated. Prolonged latent phase of labor is defined as 20 hours for the nullipara and 14 hours for the multiparous women. Cervical dilation of 3-5 cm or more plus uterine contractions represents threshold of active labor (2).

Many in health care are tempted to hospitalize women in latent phase in order to reduce mother's pain and fear. While early hospitalization of parturient women raises the risk of various complications such as increased risk of cesarean delivery (4). Findings of a study by Gifford et al. on 2447 pregnant women indicated that a prolonged latent phase is one of the leading causes of cesarean unplanned section. In $68 \%$ of the patients participated in our study

Received 11 January 2014, Revised 19 March 2014, Accepted 2 April 2014, Available online 14 April 2014

${ }^{1}$ Department of Midwifery, Tabriz Islamic Azad University, Tabriz Branch, Tabriz, Iran

${ }^{2}$ Department of Midwifery, Faculty of Nursing and Midwifery, Tabriz University of Medical Sciences, Tabriz, Iran

${ }^{3}$ Department of Physiology, Tabriz University of Medical Sciences, Tabriz, Iran

*Corresponding Author: Fahimeh Sehhatie Shafaie, Department of Midwifery, Faculty of Nursing and Midwifery, Tabriz University of Medical Sciences, Tabriz, Iran. Tel: +989143014293, Email: sehhatief@ tbzmed.ac.ir 
failure to progress in labor had led to cesarean delivery (5). Due to prolonged time intervals and length of stay hospitalization during early latent phase of labor can have negative impacts on the mothers and their families but it imposes additional costs on patients, too. On the other hand as more beds will be occupied that is also economic burden for national health system. Then due to work pressure the obstetricians may decide more than ever to perform cesarean section (6). However, most of the experts and managers of the health centers believe that postponing hospitalization of parturient women can help to prevent premature admission to labor units, avoiding unnecessary interventions in labor process and reducing costs. This is true for the cases in latent phase when no significant complication is diagnosed such as premature rupture of membrane, fetal distress or vaginal bleeding (7). Findings of a research performed by Gharoro et al. on 3130 women in labor indicated early admission of the patient and prolonged latent phase as the potential causes of increased birth defects. They also suggested that the preliminary vaginal examination of the referred women should be undertaken by an experienced person presented at admission (8).

Bailit et al. in Metro Health Center in Ohio compared outcomes in women presenting in latent $(n=2697)$ and active $(n=6121)$ labor. More latent phase women were nulliparous. Women referred in latent phase had more cesarean deliveries compared to the ones who had referred in active phase (14.2\% vs. 6.7\%). Parity check revealed that in women admitted during latent phase had more active phase arrest, oxytocin use, fetal scalp $\mathrm{PH}$ testing, intrauterine pressure catheter placed, fetal scalp electrocardiogram, monitoring and also amnionitis. However there was no significant difference between these study groups in C-section rate, delivery with forceps, vacuum, neonate intubation, bleeding and postpartum infection (3). Yet existing studies do not suggest any clear patterns for management of latent phase. On the other hand, it is imprecise to ascertain the exact time of labor onset as well as determining the length of labor and latent phase. This can explain the reason for different approaches to managing the latent stage (9).

In 2006 Rahnama and colleagues performed a survey titled "Impact of early admission in labor on method of delivery". This study compared the types of delivery between two groups of women admitted in hospital: 466 low-risk nulliparous in latent phase and 329 parturient women in active phase. Findings indicated that the number of cesarean deliveries in the first group was greater than second group (65\% vs. $24.3 \%)$. The main reason for choosing $\mathrm{C}$-section type of delivery was fetal distress. But there was no significant difference among the participants on length of stay in hospital and lack of progress in delivery (10).

It is not clear exactly that what the reason is for adverse outcomes for women admitted in latent phase, whether it is for uterine dysfunction (due to long-term pain before hospitalization), a high risk of it or admission in latent phase, prolonged hospitalization and obstetric interventions predict adverse maternal and infant outcomes. Turrant and Greulich believed that the latent phase of labor is complex and not completely understood by modern science. According to these scholars studies often ignore evaluation of latent phase because determination of onset is subjective (11). Given the above, this study is conducted on pregnant women referred to Tabriz Taleghani Hospital to detect the impact of early admission in latent phase on delivery outcomes. Then we will able to provide helpful pattern for determining hospitalization time; this can be helpful in mitigation of delivery risk and also maternal and infant postpartum complications.

\section{Material and Methods}

This descriptive research was performed in Tabriz Taleghani Hospital. The study population consisted of all pregnant women admitted for vaginal childbirth.

The sample size was estimated using the formula for calculating as average in which z-value (with 95\% CL) was 1.96 and d-value was 0.05 varied based on the researcher purpose. According to the formula used sample size was 225; considering a drop-out rate of $10 \%$ total sample size required was 220. Painful and regular uterus contractions and dilation $\leq 4$ were the factors for assessment of the onset of latent phase of labor.

The required information was collected using the birth process observation technique and by interviewing mothers and studying medical records. Data were gathered using a 5-part questionnaire including (respectively): characteristics of the subjects (women's obstetric history, laboratory test results at the time of admission, vaginal examination results, assessment of uterine contractions, vital signs), partograph forms (duration of the first phase of labor, FHR, dilation, station, number of contractions, oxytocin administration, membrane status, medication), checklist for evaluating the labor second phase (duration, type of delivery, fundal pressure during delivery process, neonate Apgar, $\mathrm{PH}$ umbilical artery blood $\mathrm{PH}$, me conium stain, procedures performed on the newborn, weight of neonates, duration of stay in NICU), third phase (duration of the third phase and placental removal method), fourth phase (complications and actions during 4th stage, vital signs prior to transfer to postpartum ward). The fourth and fifth parts of the tool were respectively devoted to the results of pre-discharge examinations (duration of stay in postpartum unit, vital signs, hemoglobin and hematocrit level, vulva and perinea hematoma, vaginal bleeding after delivery urinary complications) and examinations during first 10 days after birth (episiotomy infection, endometritis, postpartum bleeding, urinary infection and deep vein thrombosis). The instrument validity was determined using content validity. To assess validity the questionnaire was sent to 10 faculty members of Tabriz University of Medical Sciences.

The study process was as follows: full term pregnancy (38-42 weeks based on accurate LMP or 8-16 weeks ultrasound), referring to hospital during the latent 
stage, vortex presentation, optimal diameter for pelvic suitable for vaginal delivery, uncomplicated and also singleton pregnancy, a normal NST at admission time. Those eligible were enrolled in the research and studied in 4 stages. First characteristics of the participants were recorded including patient's obstetrical history (number of pregnancies, deliveries and abortions, number of live and dead children, LMP, estimated date of delivery using LMP and ultrasound, vaginal delivery history), results of the tests done on admission (proteinuria, pyuria, bacteriuria and hematuria), platelet count, PT, PLT and hemoglobin and hematocrit, the results of vaginal examinations (dilation rate, effacement and station, membrane status), measuring number and duration of uterine contractions every $10 \mathrm{~min}$, and also the results of vital signs (blood pressure and body temperature). Then those in the latent phase included in the study based on vaginal examination results. In the second step after admission to labor ward procedures performed and progress of labor were assessed and recorded in partograph form. In the third stage the subjects were transferred to delivery room; the second, third and fourth stages of labor were carefully observed and recorded in the checklist. The fourth step included recording pre-discharge examination results. Finally the subjects were asked to come to hospital on the tenth day of birth for postpartum assessment. In the fifth stage the examination results of these ten days were recorded in the postpartum complication checklist. In case of failure to attend it was completed over the phone. Prior to conducting this research, authorization was obtained from Tabriz University of Medical Sciences research committee. Explaining the research aims and methods all of the participants was voluntary and they were right to withdraw from the study at any time and were also assured of confidentiality of all information. Then written consents were obtained.

Data was statistically analyzed using SPSS 13. Descriptive statistics (The frequency, percentage, mean and standard deviation) were used to summarize data.

\section{Results}

The mean age of the women admitted during latent phase was $25.08 \pm 5.12 .175(70 \%)$ of the subjects were aged from 19-28 years. $168(67.2 \%)$ were nulliparous, the number of abortion and stillborn rates were 24 (9.4\%) and 7 (2.8\%), respectively. 79 (31.6\%) had experienced one or more vaginal childbirths. Based on 8-16 week ultrasound the mean gestational age at time of admission was $28.8 \pm 11.8$. Table 1 shows gestational characteristics of the subjects admitted in latent phase of labor.

Regarding tests results on admission, mean hemoglobin/ hematocrit levels were $12.2 \pm 1.1$ and $36.9 \pm 3.2$, respectively. Urine analysis indicated that about $17.6 \%$ suffered from urinary disorders including proteinuria, bacteriuria and pyuria. Mean platelet count, PT and PTT ratios were $200214.8 \pm 53947.9,13 \pm 0.3$ and $37.8 \pm 3.3$, respectively. Most of the women had been admitted at $3 \mathrm{~cm}$ dilated. Oxytocin induction was performed in 187 (74.8\%) of the cases. Amniotomy was used in 139 ones (55.6\%). Fetal heart rate (FHR) monitoring revealed maternal arrhythmia in $6.8 \%$ of the subjects including bradycardia and tachycardia. Assessment of uterine contractions showed that $18.8 \%$ were hypotonic while using oxytocin. The summary of the review partographs of mothers hospitalized during latent phase is shown in Table 2.

Evaluating the results of the second phase of labor showed that 208 ones (83.2\%) delivered vaginally with episiotomy. Fundal pressure was performed in $38.8 \%$ of the cases with

Table 1. Obstetrics characteristics of women admitted in Latent phase

\begin{tabular}{|c|c|c|}
\hline Obstetrics characteristics & & $\begin{array}{l}\text { Latent phase } \\
\text { N (\%) }\end{array}$ \\
\hline \multirow{3}{*}{ Gravid } & 1 & $168(67.2)$ \\
\hline & 2 & $45(18)$ \\
\hline & $2>$ & $37(14.8)$ \\
\hline Mean(SD) & & $1.6 \pm 1$ \\
\hline \multirow{2}{*}{ Para } & 0,1 & $54(21.6)$ \\
\hline & $1>$ & $24(9.6)$ \\
\hline \multirow[t]{2}{*}{ Mean(SD) } & & $0.5 \pm 0.8$ \\
\hline & 0 & $226(90.4)$ \\
\hline \multirow[t]{2}{*}{ Abortion } & 1 & $20(8)$ \\
\hline & $1>$ & $4(1.4)$ \\
\hline \multirow[t]{2}{*}{ Mean(SD) } & & $0.12 \pm 0.4$ \\
\hline & 0 & $243(97.2)$ \\
\hline \multirow[t]{2}{*}{ IUFD } & 1 & $7(2.8)$ \\
\hline & $1>$ & $0(0)$ \\
\hline \multirow[t]{2}{*}{ Mean(SD) } & & $0.02 \pm 0.16$ \\
\hline & 0,1 & $55(22)$ \\
\hline \multirow[t]{2}{*}{ History of Vaginal Delivery } & 2 & $15(6)$ \\
\hline & $>2$ & $9(3.4)$ \\
\hline Mean(SD) & & $0.47 \pm 0.7$ \\
\hline
\end{tabular}

Table 2. Partograph results of women admitted in Latent phase

\begin{tabular}{lll}
\hline Partograph results & & $\begin{array}{l}\text { Latent phase } \\
\text { N (\%) }\end{array}$ \\
\hline Use of Oxytocin & & $187(25.2)$ \\
Amniotomy & & $139(55.6)$ \\
& Ampicilin & $126(50.4)$ \\
& cephazolin & $6(2.4)$ \\
Drug administration & Hyocin & $82(32.8)$ \\
& Petidin & $20(8)$ \\
& Prometazin & $64(25.6)$ \\
& Atropin & $16(6.4)$ \\
& Normal & $233(93.2)$ \\
& Bradychardia & $4(1,6)$ \\
& Tachycardia & $6(2.4)$ \\
& Variable & $7(2.8)$ \\
& Normal without oxytocin & $107(42.8)$ \\
& Normal with oxytocin & $96(38.4)$ \\
& Hypotonic with oxytocin & $47(18.8)$ \\
\hline \multirow{4}{*}{ Contractions } & &
\end{tabular}


no power to push the baby out, yet it is wrong option for childbirth. Results of the second stage of the labor in the subjects admitted in latent phase are shown in Table 3.

Characteristics of the third and fourth labor stages in women early admitted are demonstrated in Table 4.

$28(11.2 \%)$ of the subjects admitted during latent phase experienced prolonged latent phase of labor (more than 8 hours) while $54(21.6 \%)$ had prolonged active phase (cervix dilation $<1 \mathrm{~cm} / \mathrm{h}$ ). Duration of different stages of labor in

Table 3. Second stage results of women admitted in Latent phase

\begin{tabular}{|c|c|c|}
\hline Second stage & & $\begin{array}{l}\text { Latent phase } \\
\mathrm{N}(\%)\end{array}$ \\
\hline \multirow{6}{*}{ Type of delivery } & $\begin{array}{l}\text { Vaginal and without } \\
\text { Episiotomy }\end{array}$ & $5(2)$ \\
\hline & Vaginal with Episiotomy & $208(83.2)$ \\
\hline & $\begin{array}{l}\text { Vaginal with rupture of } \\
\text { Perineum }\end{array}$ & $10(4)$ \\
\hline & Vaginal and without & \\
\hline & $\begin{array}{l}\text { Episiotomy and without } \\
\text { with rupture of Perineum }\end{array}$ & $25(10)$ \\
\hline & $\begin{array}{l}\text { Vaginal with Episiotomy and } \\
\text { Vacuum }\end{array}$ & $2(0.8)$ \\
\hline \multirow{3}{*}{$\begin{array}{l}\text { Pressure on fundus } \\
\text { in labor }\end{array}$} & Yes & $97(38.8)$ \\
\hline & No & $153(61.2)$ \\
\hline & $<5$ & $1(0.4)$ \\
\hline \multirow[t]{2}{*}{ APGAR in $1 \mathrm{~min}$} & $6-8$ & $12(4.8)$ \\
\hline & $9-10$ & $237(94.8)$ \\
\hline \multirow[t]{2}{*}{ Mean (SD) } & & $8.77 \pm 0.64$ \\
\hline & $7-7.2$ & $40(16)$ \\
\hline \multirow[t]{2}{*}{$\mathrm{PH}(\mathrm{ABG})$} & 7.21-7.4 & $187(74.8)$ \\
\hline & 7.41-7.6 & $23(9.2)$ \\
\hline Mean (SD) & & $7.29 \pm 0.08$ \\
\hline \multirow{3}{*}{ Meconium staining } & Yes & $25(10)$ \\
\hline & No & $225(90)$ \\
\hline & Without Need & $87(34.8)$ \\
\hline \multirow[t]{2}{*}{ Practice on Infant } & Stimulate, $\mathrm{O}_{2}$, Suction & $136(54.4)$ \\
\hline & Resuscitation & $27(10.8)$ \\
\hline
\end{tabular}

Table 4. Third and Fourth stages results of women admitted in Latent phase

\begin{tabular}{lll}
\hline $\begin{array}{l}\text { Third and Fourth } \\
\text { stages }\end{array}$ & & $\begin{array}{l}\text { Latent phase } \\
\text { N (\%) }\end{array}$ \\
\hline $\begin{array}{l}\text { Method of } \\
\text { Placenta } \\
\text { expulsion }\end{array}$ & Spontaneous & $226(90.4)$ \\
& Wanual & $24(9.6)$ \\
& Without & $0(0)$ \\
Fourth stage & Uterus Atonia & $219(87.6)$ \\
complications & Placenta retained & $9(3.6)$ \\
& 3 and 4 degree rupture of & $22(8.8)$ \\
& Perineum & $0(0)$ \\
& Uterus Massage on Abdomen & $148(59.2)$ \\
Doing practices & Hemorrhage & $32(12.8)$ \\
in Fourth stage & Methergine administration & $12(4.8)$ \\
& PGs administration & $6(2.4)$ \\
& Blood administration & $4(1.6)$ \\
\hline
\end{tabular}

women admitted during latent phase is shown in Table 5. Prior to transfer to postpartum ward the vital signs were as follows: blood pressure level $=112.68 \pm 8.8$, body temperature $=37.3 \pm 4.03$, heart rate $=80.4 \pm 14.8$ and respiration rate $=20.3 \pm 10.7$. Regarding pre-discharge examination of the women in delivery about 3.2 had vulvar and perineal hematoma while $6 \%$ were diagnosed with postpartum hem and $2 \%$ with urinary problems.

The mean hemoglobin and hematocrit levels were respectively $12.2 \pm 1.1$ and $36.9 \pm 3.2$ while they were $10.8 \pm 1.4$ and $33.1 \pm 3.9$ in pre-discharge examination. The admission value was then subtracted from the predischarge value so the average difference was found. The mean hemoglobin/hematocrit drop during hospitalization were $1.35 \pm 1.41$ and $3.8 \pm 4.1$, respectively.

The subjects were evaluated 10 days after birth. Episiotomy infection and urinary infection were detected in respectively $3.6 \%$ and $2 \%$. None of the patients developed postpartum endometritis, delayed hemorrhage or deep vein thrombosis.

\section{Discussion}

Our study evaluated obstetric complications in 250 women admitted to hospital during latent phase of labor. The results revealed that hospital admission during this stage is associated with increased labor and hospitalization duration as well as increased complication rates and need for maternal and newborn interventions. When a woman is admitted in the latent phase the rate of obstetric interventions increase because of inadequate uterine contractions; forced to induction of labor and undergo amniotomy that may lead to some adverse effects. Heidania et al. in a study showed that women admitted in early latent phase are more likely to be augmented with oxytocin

Table 5. Labor stages length in women admitted in Latent phase

\begin{tabular}{|c|c|c|}
\hline Labor stages length & & $\begin{array}{l}\text { Latent phase } \\
\mathrm{N}(\%)\end{array}$ \\
\hline \multirow{5}{*}{ Second stage length } & $1-20$ & $134(53.6)$ \\
\hline & $21-40$ & $82(32.8)$ \\
\hline & $41-60$ & $14(5.6)$ \\
\hline & $61-80$ & $12(4.8)$ \\
\hline & $81-100$ & $6(2.4)$ \\
\hline \multirow[t]{2}{*}{ Means \pm SD } & & $27.22 \pm 18.43$ \\
\hline & $1-5$ & $125(50)$ \\
\hline \multirow{3}{*}{ Third stage length (min) } & $6-10$ & $99(39.6)$ \\
\hline & $11-15$ & $22(8.8)$ \\
\hline & $16-20$ & $3(1.2)$ \\
\hline \multirow[t]{3}{*}{ Means $\pm S D$} & & $7.8 \pm 5.11$ \\
\hline & $10-20$ & $103(41.2)$ \\
\hline & $21-30$ & $113(45.2)$ \\
\hline \multirow{4}{*}{$\begin{array}{l}\text { Duration administration in } \\
\text { post partum ward (h) }\end{array}$} & $31-40$ & $2(0.8)$ \\
\hline & $41-50$ & $12(4.8)$ \\
\hline & $51-60$ & $0(0)$ \\
\hline & $61-70$ & $2(0.8)$ \\
\hline Means \pm SD & & $21.33 \pm 8.9$ \\
\hline
\end{tabular}


when compared to the ones admitted in active labor phase (12). Findings of a survey by Bailit et al. suggested that the women who were hospitalized to labor ward while were still in the latent phase are faced with higher risk of failure to progress in active stage, oxytocin augment, fetal scalp $\mathrm{PH}$ testing, using intrauterine pressure catheter and also internal fetal heart monitoring and ammonites which is consistent with the results of the present study (3).

Early admission during the latent phase increases the probability of maternal medication, postpartum hemorrhage and so decreases the rate of hemoglobin/ hematocrit in the postpartum period then will require long-term treatment for anemia. Findings of a research performed by Ajori and colleagues indicated excessive postpartum bleeding is more common in the mothers admitted during latent phase of labor (13) that is consistent with our report. Latent-phase admission is not associated with poor neonatal outcomes; however prolongation of this stage, the first min APGAR score, umbilical artery blood PH will be lowered while the procedures done on newborns will be increased. These kinds of studies questioned the validity of traditional belief that consider prolonged latent phase as benign condition (2). Ajori et al. predicted lower APGAR scores and higher me conium aspiration in prolonged latent phase (13). The results of a research by Hodnett et al. suggested that a prolonged latent stage ( $>12$ hours for nulliparous and $>6$ hours for multiparous) is associated with prolonged labor, low newborn Apgar score and need for neonatal resuscitation (14). The aim of modern midwifery is to provide a safe birth process for mother and her baby. Hospitalization in latent phase might have negative effects on patients including increased adverse events and also obstetric interventions and inpatient length of stay therefore it is necessary to determine admission appropriateness before hospitalization of the pregnant women, prior to attempting delivery and further interventions. Then it is obligatory to differentiate between false and real labor contractions (about 10\% of the pregnant women hospitalizations are for false labor). Avoiding common problems associated with early hospitalization, the patient in latent phase should be admitted only when painful contractions become regular or be accompanied by 1) rupture of membrane 2) bloody show, or 3) complete cervical effacement. On the other hand, prolonged hospitalization, experiencing its adverse impacts on herself and her baby and receiving aggressive interventions may negatively affect maternal satisfaction with vaginal delivery and also on their decision to reexperience vaginal birth in subsequent pregnancies. On the basis of the above it can be concluded that, except for high-risk pregnancies, hospitalization of women in latent phase should be avoided.

\section{Conclusion}

Based on the findings it is recommended the pregnant women should be trained about the signs of start of active stage during prenatal care visits. They also should be advised not to come to hospital at the onset of active phase of labor. They must be known that hospitalization is not required if no complications occur. This can prevent subsequent complications associated with early hospitalization. So more studies with larger sample size and in other medical centers are needed to alter the opinion that "prolonged latent phase of labor is benign condition". A community- based protocol can be prepared for management of this phase of labor and installed in healthcare centers.

\section{Ethical issues}

The study was approved by the ethic committee of Tabriz University of Medical Sciences.

\section{Conflict of interests}

Authors declare that there are no any conflict of interests.

\section{Acknowledgments}

The authors would like to thank the director and staff of Tabriz Taleghani Hospital. This work has been funded by Research Vice-Chancellor of Tabriz University of Medical Sciences.

\section{References}

1. Neal JL, Lowe NK, Ahijevych KL, Patrick TE, Cabbage LA, Corwin EJ. "Active labor" duration and dilation rates among low-risk, nulliparous women with spontaneous labor onset: a systematic review. J Midwifery Womens Health 2010; 55: 308-18.

2. Cunningham FG, Leveno KJ, Bloom SL, Hauth JC, Rouse DJ, Spong CY. Williams Obstetrics. 23st Ed. New York: McGraw Hill; 2010.

3. Bailit JL, Dierker L, Blanchard MH, Mercer BM. Outcomes of women presenting in active versus latent phase of spontaneous labor. Obstet Gynecol 2005;105:77-9.

4. Impey L, Hobson J, O’Herlihy C. Graphic analysis of actively managed labor: Prospective computation of labor progress in 500 consecutive nulliparous women in spontaneous labor at term. Am J Obstet Gynecol 2000; 183: 438-43.

5. Gifford DS, Morton SC, Fiske M, Keesey J, Keeler E, Kahn KL. Lack of progress in labor as a reason for cesarean. Obstet Gynecol 2000; 95: 589-95.

6. Hemminki E, Simukka R. The timing of hospital admission and progress of labor. Eur J Obstet Gynecol Reprod Biol 2000; 22: 85-94.

7. Vaziri F, Gheidar F. Labor Outcomes of Nulliparous Women Presented in Latent Phase Referred to Obstetric Ward of Shariati Teaching Hospital, Bandar Abbas. Journal Dena 2010; 4: 32-9.

8. Gharoro EP, Enabudoso EJ. Labour management: an appraisal of the role of false labour and latent phase on the delivery mode. J Obstet Gynaecol 2006; 26:534-7.

9. Gross MM, Drobnic S, Keirse M. Ifluence of fixed and timedependent factors on duration of normal first stage labor. Birth 2005; 32: 27-33.

10. 10-Rahnama P, Ziaei S, Faghihzadeh S. Impact of early admission in labor on method of delivery. Int J Gynecol Obstet 2006; 92: 217-20.

11. Greulich B, Tarrant B. The latent phase of labor: diagnosis and management. J Midwifery Womens Health 2007; 52:190-8. 
12. Heydarnia MA, Rahnama P, Montazeri A, Ebadi M, Rahmati Najarkolaei F. The relationship between early admission in labor and the occurrence of obstetrical complications. Payesh Journal 2009; 7: 235- 39.

13. Ajori L, Masoumi M, Rahbari H, Ahmadi K. Maternal and neonatal complications in prolonged latent phase in women referring in Shohada Hospital in Tehran. Journal of Shaheed
Sadoughi University of Medical Sciences 2010;18: 3-7.

14. Hodnet ED, Stremler R, Willan AR, Weston JA, Lowe NK, Simpson KR. Effect on birth outcomes of formalized approach to care in hospital labor assessment units: international, randomized controlled trial. BMJ 2008; 337:a1021.

Copyright ( 2014 The Author(s); This is an open-access article distributed under the terms of the Creative Commons Attribution License (http://creativecommons.org/licenses/by/4.0), which permits unrestricted use, distribution, and reproduction in any medium, provided the original work is properly cited. 